\title{
Tactical manufacturing capacity planning based on discrete event simulation and throughput accounting: A case study of medium sized production enterprise
}

\author{
Jurczyk-Bunkowska, M. ${ }^{\mathrm{a},{ }^{*}}$ \\ aFaculty of Economics and Management, Opole University of Technology, Poland
}

\begin{abstract}
A B S T R A C T
The article presents the application of the original methodology to support tactical capacity planning in a medium-sized manufacturing company. Its essence is to support medium-term decisions regarding the development of the production system through economic assessment of potential change scenarios. It has been assumed that the developed methodology should be adapted to small and medium-sized enterprises (SMEs). Due to their flexibility, they usually have limited time for decision-making, and due to limited financial resources, they rely on internal competencies. The proposed approach that does not require mastery of mathematical modelling but allows streamlining capacity planning decisions. It uses the reasoning of throughput accounting (TA) supported by data obtained based on discrete event simulation (DES). Using these related tools in the design and analysis of change scenarios, make it possible for SME managers to make a rational decision regarding the development of the production system. Case studies conducted in a roof window manufacturing company showed the methodology. The application example presented in the article includes seven change scenarios analyzed based on computer simulations by the software Tecnomatix Plant Simulation. The implementation of the approach under real conditions has shown that a rational decision-making process is possible over time scale and with the resources available to SMEs for this type of decision.
\end{abstract}

\author{
ARTICLE INFO \\ Keywords: \\ Decision process; \\ Capacity planning; \\ Discrete event simulation (DES); \\ Throughput accounting (TA); \\ Plant simulation; \\ Small and medium-sized \\ enterprises (SME); \\ Production scenarios; \\ Tecnomatix Plant Simulation \\ *Corresponding author: \\ m.jurczyk-bunkowska@po.edu.pl \\ (Jurczyk-Bunkowska, M.)
}

Article history:

Received 7 June 2021

Revised 12 October 2021

Accepted 13 October 2021

\section{(1)}

Content from this work may be used under the terms of the Creative Commons Attribution 4.0 International Licence (CC BY 4.0). Any further distribution of this work must maintain attribution to the author(s) and the title of the work, journal citation and DOI.

\section{References}

[1] Seyedan, M., Mafakheri, F. (2020). Predictive big data analytics for supply chain demand forecasting: Methods, applications, and research opportunities, Journal of Big Data, Vol. 7, Article No. 53, doi: 10.1186/s40537-02000329-2.

[2] Kampa, A., Gołda, G., Paprocka, I. (2017). Discrete event simulation method as a tool for improvement of manufacturing systems, Computers, Vol. 6, No. 1, Article No. 10, doi: 10.3390/computers6010010.

[3] Patalas-Maliszewska, J., Topczak, M. (2021). A new management approach based on additive manufacturing technologies and Industry 4.0 requirements, Advances in Production Engineering \& Management, Vol. 16, No. 1, 125-135, doi: 10.14743/apem2021.1.389.

[4] Sabet, E., Yazdani, B., Kian, R., Galanakis, K. (2020). A strategic and global manufacturing capacity management optimisation model: A scenario-based multi-stage stochastic programming approach, Omega, Vol. 93, Article No. 102026, doi: 10.1016/j.omega.2019.01.004.

[5] Jacobs, F.R., Chase, R.B. (2008). Operations and supply management: The core, McGraw Hill/Irwin, New York, USA.

[6] Zhang, R.-Q. (2007). Research on capacity planning under stochastic production and uncertain demand, Systems Engineering - Theory \& Practice, Vol. 27, No. 1, 51-59, doi: 10.1016/S1874-8651(08)60006-X. 
[7] Gallego-García, S., Reschke, J., García-García, M. (2019). Design and simulation of a capacity management model using a digital twin approach based on the viable system model: Case study of an automotive plant, Applied Sciences, Vol. 9, No. 24, Article No. 5567, doi: 10.3390/app9245567.

[8] Sawik, T. (2009). Multi-objective due-date setting in a make-to-order environment, International Journal of Production Research, Vol. 47, No. 22, 6205-6231, doi: 10.1080/00207540902810585.

[9] Alp, 0., Tan, T. (2008). Tactical capacity management under capacity flexibility, IIE Transactions, Vol. 40, No. 3, 221-237, doi: 10.1080/07408170701488052.

[10] Chen, Y.-Y., Chen, T.-L., Liou, C.-D. (2012). Medium-term multi-plant capacity planning problems considering auxiliary tools for the semiconductor foundry, The International Journal of Advanced Manufacturing Technology, Vol. 64, No. 9-12, 1213-1230, doi: 10.1007/s00170-012-4080-9.

[11] Tavaghof-Gigloo, D., Minner, S., Silbermayr, L. (2016). Mixed integer linear programming formulation for flexibility instruments in capacity planning problems, Computers \& Industrial Engineering, Vol. 97, 101-110, doi: 10.1016/j.cie.2016.04.013.

[12] Zschorn, L., Müller, S., Ivanov, D. (2017). Capacity planning on key work stations in a hybrid MTO-ETO production system: A case-study on Siemens AG, International Journal of Inventory Research, Vol. 4, No. 2-3, 214-232, doi: $10.1504 /$ ijir.2017.088743.

[13] Fleischmann, B., Ferber, S., Henrich, P. (2006). Strategic planning of BMW's global production network, INFORMS Journal on Applied Analytics, Vol. 36, No. 3, 194-208, doi: 10.1287/inte.1050.0187.

[14] Yu, V.F., Normasari, N.M E., Luong, H.T. (2015). Integrated location-production-distribution planning in a multiproducts supply chain network design model, Mathematical Problems in Engineering, Vol. 2015, Article ID 473172, doi: $10.1155 / 2015 / 473172$.

[15] Lin, J.T., Chen, T.-L., Chu, H.-C. (2014). A stochastic dynamic programming approach for multi-site capacity planning in TFT-LCD manufacturing under demand uncertainty, International Journal of Production Economics, Vol. 148, 21-36, doi: 10.1016/j.ijpe.2013.11.003.

[16] Wang, K.-J., Nguyen, P.H. (2017). Capacity planning with technology replacement by stochastic dynamic programming, European Journal of Operational Research, Vol. 260, No. 2, 739-750, doi: 10.1016/j.ejor.2016.12.046.

[17] Geng, N., Jiang, Z. (2009). A review on strategic capacity planning for the semiconductor manufacturing industry, International Journal of Production Research, Vol. 47, No.13, 3639-3655, doi: 10.1080/00207540701871051.

[18] Teerasoponpong, S., Sopadang, A. (2021). A simulation-optimization approach for adaptive manufacturing capacity planning in small and medium-sized enterprises, Expert Systems with Applications, Vol. 168, Article No. 114451, doi: 10.1016/j.eswa.2020.114451.

[19] Renna, P. (2016). A decision investment model to design manufacturing systems based on a genetic algorithm and Monte-Carlo simulation, International Journal of Computer Integrated Manufacturing, Vol. 30, No. 6, 590-605, doi: 10.1080/0951192x.2016.1187299.

[20] Huka, M.A., Grenzfurtner, W., Zauner, B., Gronalt, M. (2021). Capacity planning of a mixed-model assembly line for prefabricated housebuilding elements, Procedia Computer Science, Vol. 180, 706-713, doi: 10.1016/j.procs. 2021.01.293.

[21] Zhang, B.-X., Song, J., Yu, X.-L. (2012). Research about short-term production capacity decision-making, Physics Procedia, Vol. 24, Part B, 1207-1213, doi: 10.1016/j.phpro.2012.02.180.

[22] Coleman, S., Göb, R., Manco, G., Pievatolo, A., Tort-Martorell, X., Reis, M.S. (2016). How can SMEs benefit from big data? Challenges and a path forward, Quality and Reliability Engineering International, Vol. 32, No. 6, 2151-2164, doi: $10.1002 /$ qre.2008.

[23] Straka, M., Khouri, S., Lenort, R., Besta, P. (2020). Improvement of logistics in manufacturing system by the use of simulation modelling: A real industrial case study, Advances in Production Engineering \& Management, Vol. 15, No. 1, 18-30, doi: 10.14743/apem2020.1.346.

[24] Kotevski, Ž., Jovanoski, B., Minovski, R. (2015). Simulation model for improved production planning and control through quality, cycle time and batch size management, Journal of Engineering Management and Competitiveness, Vol. 5, No. 1, 40-45, doi: 10.5937/jemc1501040k.

[25] Turk, M., Pipan, M., Šimic, M., Herakovič, N. (2020). Simulation-based time evaluation of basic manual assembly tasks, Advances in Production Engineering \& Management, Vol. 15, No. 3, 331-344, doi: 10.14743/apem2020.3. $\underline{369}$.

[26] Bangsow, S. (2010). Manufacturing simulation with plant simulation and simtalk: Usage and programming with examples and solutions, Springer, Berlin, Germany, doi: 10.1007/978-3-642-05074-9.

[27] Zhang, L., Zhou, L., Ren, L., Laili, Y. (2019). Modeling and simulation in intelligent manufacturing, Computers in Industry, Vol. 112, Article No. 103123, doi: 10.1016/i.compind.2019.08.004.

[28] Gansterer, M., Almeder, C., Hartl, R.F. (2014). Simulation-based optimization methods for setting production planning parameters, International Journal of Production Economics, Vol. 151, 206-213, doi: 10.1016/j.ijpe.2013. $\underline{10.016}$.

[29] Gyulai, D., Monostori, L. (2017). Capacity management of modular assembly systems, Journal of Manufacturing Systems, Vol. 43, Part 1, 88-99, doi: 10.1016/i.jmsy.2017.02.008.

[30] Bae, K.-H., Jones, M., Evans, G., Antimisiaris, D. (2019). Simulation modelling of patient flow and capacity planning for regional long-term care needs: A case study, Health Systems, Vol. 8, No. 1, 1-16, doi: 10.1080/20476965. 2017.1405873.

[31] Antonelli, D., Litwin, P., Stadnicka, D. (2018). Multiple system dynamics and discrete event simulation for manufacturing system performance evaluation, Procedia CIRP, Vol. 78, 178-183, doi: 10.1016/j.procir.2018.08.312.

[32] Sarda, A., Digalwar, A.K. (2018). Performance analysis of vehicle assembly line using discrete event simulation modelling, International Journal of Business Excellence, Vol. 14, No. 2, 240-255, doi: 10.1504/ijbex.2018. 10009766. 
[33] Lang, S., Reggelin, T., Müller, M., Nahhas, A. (2021). Open-source discrete-event simulation software for applications in production and logistics: An alternative to commercial tools?, Procedia Computer Science, Vol. 180, 978987, doi: 10.1016/i.procs.2021.01.349.

[34] Istokovic, D., Perinic, M., Dobovicek, S., Bazina, T. (2019). Simulation framework for determining the order and size of the product batches in the flow shop: A case study, Advances in Production Engineering \& Management, Vol. 14, No. 2, 166-176, doi: 10.14743/apem2019.2.319.

[35] Carvalho, A.N., Scavarda, L.F., Oliveira, F. (2017). An optimisation approach for capacity planning: Modelling insights and empirical findings from a tactical perspective, Production, Vol. 27, Article No. e20170014, doi: $\underline{10.1590 / 0103-6513.001417 .}$

[36] Yin, R.K. (2003). Case study research: Design and methods, Third Edition, Sage PublicationsThousand Oaks, USA.

[37] Voss, C., Tsikriktsis, N., Frohlich, M., (2002). Case research in operations management, International Journal of Operations \& Production Management, Vol. 22, No. 2, 195-219, doi: 10.1108/01443570210414329.

[38] Urban, W. (2019). TOC implementation in a medium-scale manufacturing system with diverse product rooting, Production \& Manufacturing Research, Vol. 7, No. 1, 178-194, doi: 10.1080/21693277.2019.1616002.

[39] Baxendale, S.J., Raju, P.S. (2004). Using ABC to enhance throughput accounting: A strategic perspective, Cost Management, Vol. 1-2, 31-38.

[40] Corbett, T. (1998). Throughput accounting: TOC's management accounting system, North River Press, Great Barrington, USA.

[41] Dražić Lutilsky, I., Liović, D., Marković, M. (2018). Throughput accounting: Profit-focused cost accounting method, In: Proceedings of International Conference Interdisciplinary Management Research XIV, Opatija, Croatia, 1382-1395.

[42] Figueira, G., Almada-Lobo, B. (2014). Hybrid simulation-optimization methods: A taxonomy and discussion, Simulation Modelling Practice and Theory, Vol. 46, 118-134, doi: 10.1016/j.simpat.2014.03.007.

[43] Aggogeri, F., Faglia, R., Mazzola, M., Merlo, A. (2015). Automating the simulation of SME processes through a discrete event parametric model, International Journal of Engineering Business Management, Vol. 7, 4 pages, doi: 10.5772/59958.

[44] Kim, S., Cox, J.F., \& Mabin, V.J. (2010). An exploratory study of protective inventory in a re-entrant line with protective capacity, International Journal of Production Research, Vol. 48, No. 14, 4153-4178, doi: 10.1080/002075 40902991666. 


\section{APEM}

Advances in Production Engineering \& Management

Letnik 16 | Številka 3 | September 2021 | Strani 335-347

https://doi.org/10.14743/apem2021.3.404
ISSN 1854-6250

Spletna stran: apem-journal.org Izvirni znanstveni članek

\title{
Taktično načrtovanje proizvodnih zmogljivosti na podlagi simulacije diskretnih dogodkov in preverjanja pretočnosti: Študija primera srednje velikega proizvodnega podjetja
}

\author{
Jurczyk-Bunkowska, M. ${ }^{\mathrm{a}, *}$ \\ aFaculty of Economics and Management, Opole University of Technology, Poland
}

\section{POVZETEK}

V članku je predstavljena uporaba izvirne metodologije za podporo taktičnega načrtovanja zmogljivosti $\mathrm{v}$ srednje velikem proizvodnem podjetju. Njeno bistvo je podpirati srednjeročne odločitve glede razvoja proizvodnega sistema z ekonomsko presojo možnih scenarijev sprememb. Predpostavljeno je bilo, da bo razvita metodologija prilagojena malim in srednje velikim podjetjem (SMEs). Zaradi svoje prilagodljivosti imajo običajno omejen čas za odločanje, zaradi omejenih finančnih sredstev pa se zanašajo na notranje kompetence. Predlagani pristop ne zahteva obvladovanja matematičnega modeliranja, ampak omogoča racionalizacijo odločitev pri načrtovanju zmogljivosti. Uporablja sklepanje na podlagi preverjanja pretočnosti (TA), s podporo podatkov, pridobljenih na podlagi simulacije diskretnih dogodkov (DES). Z uporabo teh povezanih orodij pri načrtovanju in analizi scenarijev sprememb omogoča menedžerjem SME, da sprejmejo racionalno odločitev glede razvoja proizvodnega sistema. Metodologija je predstavljena na študijah primerov, izvedenih v podjetju za proizvodnjo strešnih oken. Primer uporabe, predstavljen v članku, vključuje sedem scenarijev sprememb, analiziranih na podlagi računalniških simulacij s programsko opremo Tecnomatix Plant Simulation. Implementacija pristopa v realnih razmerah je pokazala, da je racionalen proces odločanja mogoče izvesti v časovnem obsegu in s sredstvi, ki so SME na voljo za tovrstne odločitve.

\section{PODATKI O ČLANKU}

Ključne besede:

Postopek odločanja;

Načrtovanje zmogljivosti;

Simulacija diskretnih dogodkov

(DES);

Preverjanje pretočnosti (TA);

Simulacija tovarne;

Majhne in srednje velike

podjetja (SME);

Proizvodni scenariji;

Tecnomatix Plant Simulation

*Kontaktna oseba:

m.jurczyk-bunkowska@po.edu.pl

(Jurczyk-Bunkowska, M.)

Zgodovina članka:

Prejet 7. junija 2021

Popravljen 12. oktobra 2021

Sprejet 13. oktobra 2021

\section{(i)}

Content from this work may be used under the terms of the Creative Commons Attribution 4.0 International Licence (CC BY 4.0). Any further distribution of this work must maintain attribution to the author(s) and the title of the work, journal citation and DOI. 